\title{
Retraction Note to: A new synthetic derivative of cryptotanshinone KYZ3 as STAT3 inhibitor for triple-negative breast cancer therapy
}

Wenda Zhang, Wenying Yu, Guiping Cai, Jiawen Zhu, Chao Zhang, Shanshan Li, Jianpeng Guo, Guoping Yin, Chen Chen and Lingyi Kong

Retraction Note to: Cell Death \& Disease https://doi.org/10.1038/s41419-018-1139-z published online 27 October 2018

The Editors have retracted this article. After publication concerns were raised about two of the figures. Specifically:

- In Figure 3B there appear to be areas of overlap and duplication

- In Figure 6C the HE T1 and HE T2 panels appear to be the same
The Editors therefore no longer have confidence in the data presented. Wenda Zhang, Wenying Yu, Guiping Cai, Jiawen Zhu, Shanshan Li, Jianpeng Guo, Guoping Yin, Chen Chen and Lingyi Kong disagree with this retraction. Chao Zhang has not responded to any correspondence from the Editors or the Publisher about this retraction.

Published online: 21 May 2021 